\title{
Laënnec HURBON
}

docteur en Théologie (Institut catholique de Paris) et en Sociologie (Sorbonne), directeur de recherche au CNRS et professeur à I'Université Quisqueya de Port-au-Prince

\section{(2007)}

\section{"La révolution haïtienne:}

\section{une avancée postcoloniale".}

Un document produit en version numérique par Jean-Marie Tremblay, bénévole, professeur de sociologie au Cégep de Chicoutimi

Courriel: jean-marie tremblay@uqac.ca

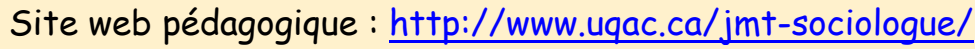

Dans le cadre de: "Les classiques des sciences sociales" Une bibliothèque numérique fondée et dirigée par Jean-Marie Tremblay, professeur de sociologie au Cégep de Chicoutimi

Site web: http://classiques.uqac.ca/

Une collection développée en collaboration avec la Bibliothèque

Paul-Émile-Boulet de I'Université du Québec à Chicoutimi Site web: http://bibliotheque.uqac.ca/ 


\section{Politique d'utilisation de la bibliothèque des Classiques}

Toute reproduction et rediffusion de nos fichiers est interdite, même avec la mention de leur provenance, sans l'autorisation formelle, écrite, du fondateur des Classiques des sciences sociales, Jean-Marie Tremblay, sociologue.

Les fichiers des Classiques des sciences sociales ne peuvent sans autorisation formelle:

- être hébergés (en fichier ou page web, en totalité ou en partie) sur un serveur autre que celui des Classiques.

- servir de base de travail à un autre fichier modifié ensuite par tout autre moyen (couleur, police, mise en page, extraits, support, etc...),

Les fichiers (.html, .doc, .pdf, .rtf, .jpg, .gif) disponibles sur le site Les Classiques des sciences sociales sont la propriété des Classiques des sciences sociales, un organisme à but non lucratif composé exclusivement de bénévoles.

Ils sont disponibles pour une utilisation intellectuelle et personnelle et, en aucun cas, commerciale. Toute utilisation à des fins commerciales des fichiers sur ce site est strictement interdite et toute rediffusion est également strictement interdite.

\section{L'accès à notre travail est libre et gratuit à tous les utilisa- teurs. C'est notre mission.}

Jean-Marie Tremblay, sociologue

Fondateur et Président-directeur général, LES CLASSIQUES DES SCIENCES SOCIALES. 
Cette édition électronique a été réalisée par Jean-Marie Tremblay, bénévole, professeur de sociologie au Cégep de Chicoutimi à partir de :

\section{Laënnec HURBON}

\section{"La révolution haïtienne: une avancée postcoloniale."}

Un texte publié dans la revue Rue Descartes, vol. 4, no 58, 2007, pp. 56-66. Paris : Les Presses universitaires de France.

[Autorisation formelle accordée par l'auteur le 19 mai 2009 de diffuser toutes ses publications dans Les Classiques des sciences sociales.]

Courriel : Ihurbon@yahoo.com

Polices de caractères utilisée: Comic Sans, 12 points.

Édition électronique réalisée avec le traitement de textes Microsoft Word 2008 pour Macintosh.

Mise en page sur papier format : LETTRE (US letter), $8.5^{\prime \prime} \times 11^{\prime \prime}$ )

Édition numérique réalisée le 2 octobre 2010 à Chicoutimi, Ville de Saguenay, province de Québec, Canada.

\section{Fait avec}

Macintosh 


\section{Laënnec HURBON}

docteur en Théologie (Institut catholique de Paris) et en Sociologie (Sorbonne),

directeur de recherche au CNRS et professeur

à l'Université Quisqueya de Port-au-Prince Doyen

\section{"La révolution haïtienne: une avancée postcoloniale."}

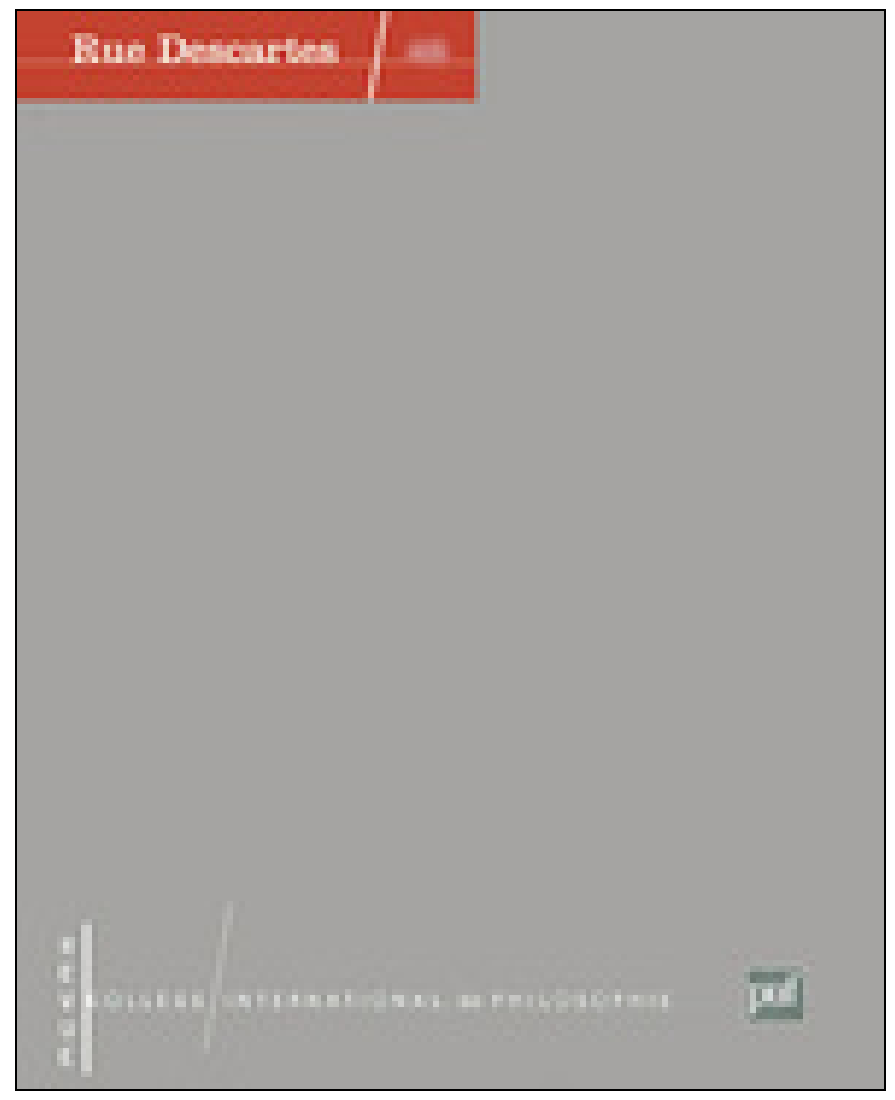

Un texte publié dans la revue Rue Descartes, vol. 4, no 58, 2007, pp. 56-66. Paris : Les Presses universitaires de France. 


\section{Table des matières}

\section{Introduction}

- Spécificité de la révolution haïtienne

- La nature de l'esclavage outre-Atlantique

- Les difficultés du nouvel État indépendant

- La révolution haïtienne et la sortie de la vision coloniale de l'histoire 
[56]

\author{
Laënnec HURBON
}

"La révolution haïtienne: une avancée postcoloniale".

Un texte publié dans la revue Rue Descartes, vol. 4, no 58, 2007, pp. 56-66. Paris: Les Presses universitaires de France.

\title{
INTRODUCTION
}

\section{Retour à la table des matières}

L'insurrection des esclaves de Saint Domingue dans la nuit du 22 au 23 août $1791{ }^{1}$ a été un événement capital dans l'histoire universelle, elle aboutit en 1804 à l'indépendance d'Haïti après une victoire de l'armée des indigènes contre les armées napoléoniennes fortes d'environ 50000 hommes expédiés sous la direction du général Leclerc. Ce fut une véritable révolution, mais elle n'a pas été perçue comme telle dans l'historiographie française et européenne. Bien plus, elle a été systématiquement banalisée. Or elle suscite un nombre considérable d'interrogations pour lesquelles jusqu'à présent nous ne disposons pas de réponses satisfaisantes.

1 Voir L. Hurbon (sd.), L'Insurrection des esclaves de Saint Domingue (22 au 23 août 1791), Karthala, Paris, 2000 ; voir aussi C.R.L. James, Les Jacobins noirs. Toussaint Louverture et la révolution de Saint Domingue, [1938], Gallimard [1949], Éd. caribéennes, Paris, 1983 ; M. Dorigny (sd), Haïti Première république noire, Publications de la société française d'histoire outre-mer et association pour l'étude de la colonisation européenne, Paris, 2003. 
D'abord, il faudra savoir quelle interprétation on a fait en Europe, en Amérique latine et aux États-Unis de la révolution haïtienne. De même on devra s'enquérir de la place qui est faite à cette révolution dans l'histoire et l'anthropologie naissantes au XIXe siècle. Que deviennent les notions de race, de nation, de religion dans le cadre du nouvel État non européen qui s'établit dans la Caraïbe? Quelles sont les contraintes rencontrées par ce nouvel État issu d'une classe d'esclaves révoltés? Dans la littérature européenne comme dans la philosophie, comment parvient-on à penser les rapports entre l'universalisme des Lumières et la particularité des cultures autres (non-occidentales) mais matinées du triple héritage européen, amérindien et africain? Quelle vision de l'Afrique la révolution haïtienne impose-t-elle? Et quelle mémoire aujourd'hui induit la révolution haïtienne pour l'histoire universelle? Nous n'avons pas la prétention d'aborder toutes ces questions posées en vrac autour de la révolution haiitienne. Nous espérons seulement susciter quelque curiosité philosophique sur la signification de la révolution haïtienne et plus précisément sur l'avancée qu'elle représente dans les controverses actuelles autour de la postcolonialité. Nous nous bornerons à une réflexion sur [57] les problèmes suivants : la spécificité de la révolution haïtienne, la nature de l'esclavage révélée par la révolution haïtienne, les obstacles rencontrés au niveau national et international par le nouvel État indépendant et enfin le caractère universel de la révolution haïtienne et la sortie d'une vision coloniale de l'histoire.

\section{Spécificité de la révolution haïtienne}


Tout d'abord, il n'est pas inutile de rappeler en quoi une véritable révolution ${ }^{2}$ s'est produite à Saint Domingue-Haïti et en quoi elle se différencie de la révolution américaine et de la révolution française. La révolution américaine, tout en ayant des sources communes avec la révolution française, cherche surtout à limiter le pouvoir des gouvernants en mettant en avant les droits des individus, puis en créant des contre-pouvoirs selon un système qu'on appelle check and balance. Les droits de l'homme ne sont pas ici l'obsession, alors que pour la révolution française, la liberté et la propriété sont considérées comme des prédicats de l'homme lui-même qui dispose ainsi de droits considérables et inaliénables. Dans cette perspective, le droit est fondé dans l'homme et non pas dans la nature ni dans une «surnature », c'est pour cela que la révolution française fera appel constamment à la raison et devra assurer la défense des droits de l'homme à partir du pouvoir politique. Aux sources de ces deux révolutions 3 , il y a le travail accompli par les Lumières et leurs principaux représentants (Voltaire, Rousseau, Diderot, Montesquieu, Diderot ou d'Alembert).

Avec le principe énoncé par Rousseau : «tous les hommes naissent libres et égaux en droit », c'est bien une nouvelle époque de l'histoire de l'humanité universelle qui s'ouvre, plus aucune forme de domination d'un homme sur un autre ne peut être légitimée, l'ordre social selon lequel fonctionnait la France va être bouleversé par la déclaration universelle des droits de l'homme et du citoyen. Mais le changement se produit dans l'idée, au niveau de la manière de concevoir l'ordre social, le monde et l'homme. C'est pour cela que Hegel parlera d'un « superbe lever de soleil ». De là, philosophes et historiens de la révolution française auront tendance à faire de la révolution française le point de

2 Nous reprenons ici les éléments principaux d'un article sur « Actualité et universalité de la révolution haïtienne » que nous avons publié en collaboration avec $\mathrm{Mi}$ chel Hector dans la revue franco-haïtienne Conjonction en 2005.

3 Pour une approche comparative des révolutions française et américaine sur les Droits de l'homme, voir l'article de Jean François Kervégan, «Les droits de I'homme » dans Notions de philosophie, D. Kambouchner (sd), Gallimard, Folio Essais, Paris, 1996, p. 637-696. Mais l'auteur ne parle pas de la révolution haïtienne. 
départ d'un universalisme conquérant qui considérera les autres sociétés comme des sociétés devant un jour parvenir au stade auquel sont parvenues la France ou l'Europe. La déclaration des droits de l'Homme vaut essentiellement pour l'Occidental en général, dont la civilisation va se déployer en intégrant [58] progressivement les autres sociétés qui connaîtraient un retard par rapport à la grande et vraie histoire : celle du monde occidental. C'est une telle vision qui sous-tend la difficulté de reconnaître en Europe la spécificité de la révolution haïtienne. Cette vision s'enracine dans les limites de la philosophie des Lumières au niveau de la manière de considérer la définition de l'homme. L'homme de la déclaration des droits... est conçu comme l'homme occidental dont les paramètres se reconnaissent dans la langue, la religion, le type d'organisation familiale, la raison etc. Sur cette base, on décidera que les esclaves sont incapables de penser leur liberté, il suffira que la révolution des esclaves - quand elle est reconnue - soit interprétée comme un complément, un achèvement de la révolution française. L'esclave noir doit son état à son niveau culturel qui impliquerait une certaine proximité avec la nature; l'esclavage est tenu alors pour une discipline pédagogique qui amènera peu à peu l'Africain à la condition de civilisé, c'est-à-dire d'un être humain à part entière. L'abolition de l'esclavage sera pour cela graduelle. Or la révolution haitienne va bousculer toutes ces idées de type raciste et ouvrira pour la première fois une nouvelle époque de l'histoire pour l'ensemble des peuples non occidentaux, dont ceux qui ont été placés en esclavage, ou sous domination coloniale. Nous pouvons maintenant rapidement souligner les aspects clés de la révolution haïtienne.

L'antiracisme: alors que l'idéologie qui présidait à l'esclavage faisait du «nègre » un être incapable de s'élever au sens de la liberté parce qu'il serait infantile, primitif ou barbare, l'insurrection des esclaves à Saint Domingue et l'indépendance sont alors une démonstration des capacités des Noirs à se battre pour la liberté, à mettre donc leur vie en jeu pour cette valeur qu'est la liberté. Dans la première Constitution du pays en 1805, la hiérarchie raciale sur laquelle était bâti le système esclavagiste est immédiatement contestée: «Toute accep- 
tion de couleur parmi les enfants d'une seule et même famille, dont le chef de l'État est le père, devant nécessairement cesser, les Haïtiens ne seront désormais connus que sous la dénomination générique de Noirs. »

L'anticolonialisme: à la proclamation de l'indépendance en 1804, le nom indien d'Ayiti est repris et il est interdit aux Français d'être propriétaires, pendant que la nationalité haïtienne est attribuée aux soldats Polonais de l'expédition napoléonienne, qui ont rejoint le camp de l'armée des « indigènes».

L'anti-esclavagisme: le processus révolutionnaire est enclenché à partir de l'insurrection générale des esclaves; de nombreuses révoltes avaient eu lieu auparavant, mais c'est celle de 1791 qui représente la première révolte victorieuse d'esclaves qui aboutira à la création d'un État indépendant. La première constitution déclare que tout Africain qui touche le sol d'Haïti est [59] automatiquement libre. Au milieu d'un environnement hostile, puisque tous les autres pays de la Caraïbe sont encore au début du XIX siècle sous le joug de l'esclavage, les premiers chefs d'État (dont d'abord Toussaint Louverture, gouverneur de Saint Domingue, avec la Constitution de 1801) démontrent que l'indépendance est fondée sur le refus systématique de l'institution esclavagiste. Certains historiens ajoutent même que le refus de l'esclavage va jusqu'à la contestation du système de l'économie de plantation, car les nouveaux paysans haïtiens choisissent d'éviter quand ils le peuvent la production des denrées d'exportation.

\section{La nature de l'esclavage outre-Atlantique}

\section{Retour à la table des matières}

Pour saisir toute la signification de la révolution haïtienne, il convient tout d'abord de se rappeler la nature de l'esclavage outreAtlantique. C'est tout un continent qui a été voué à l'esclavage par la Traite, un groupe précis d'êtres humains est retranché de la condition 
humaine ordinaire. Presque tous les pays de l'Europe participent à l'organisation de ce système inique et en profitent d'une manière ou d'une autre à travers l'économie de plantation qui représente un système de concentration à ciel ouvert. Pendant à peu près quatre siècles, Traite et esclavage feront l'enrichissement de l'Europe. Mais là ne réside pas encore toute l'horreur de l'esclavage outre-Atlantique, ni sa spécificité véritable. L'esclave déporté d'Afrique doit passer par une amnésie culturelle pour être rivé à son statut d'esclave, c'est-à-dire à celui d'un être nu, dénudé, sans prédécesseurs, sans descendants, sans origine, donc d'un être mort socialement pour reprendre la définition de l'esclave proposée par Orlando Patterson.

L'esclavage représente un événement capital - et non pas accidentel - dans la constitution de la modernité occidentale, comme le soutiennent Paul Gilroy ${ }^{4}$ et Stephan Palmié 5 . Il ne peut être évalué sur la seule base des avantages économiques tirés sur la pratique. Il est certes en continuité avec l'événement de la Conquête qui a produit le désastre génocidaire indien, mais il est quelque part en rupture 6 avec les traites musulmanes et l'esclavage africain (même si l'esclavage est toujours de même nature partout où il est pratiqué) pour une raison bien particulière: si auparavant dans l'Antiquité gréco-romaine, au Moyen Âge et dans l'Afrique [60] précoloniale, l'esclave était toujours considéré comme un étranger, il n'était pas contraint d'abandonner son système de croyances et sa culture. Cette fois, à partir du XVI siè-

4 P. Gilroy, L'Atlantique noir. Modernité et double conscience, Kargo, Paris, 2003 (1e éd., The black Atlantic, Harvard University Press, Cambridge, 1993).

5 S. Palmié, Wizards and Scientists, Explorations in afro-cuban Modernity and Tradition, Duke University Press, Durham and London, 2002.

6 Voir les critiques pertinentes adressées par Christine Chivallon (« Débat sur une relecture de l'histoire de la Traite négrière », avec $B$. Vincent et $D$. Roche, dans La revue d'histoire moderne \& contemporaine, supplément 2005 n`52-4 bis, p.4653) à l'ouvrage d'Olivier Pétré-Grenouilleau (Les Traites négrières. Essai d'histoire globale, Gallimard, Paris, 2004) : on trouve dans cet important ouvrage sur les Traites une confusion entre Traite et commerce colonial, un isolement de la Traite des systèmes esclavagistes, une faible part de l'Europe dans la pratique déjà vieille de la Traite, et enfin trop peu d'importance accordée à la formation de sociétés racialisées qui sont une conséquence de la Traite outre-Atlantique. 
cle, le monde européen se sait investi d'une mission universelle qui le conduit - grâce au christianisme et à travers lui - à prétendre viser l'assimilation de l'esclave à sa culture, pendant que cette assimilation devra rester à l'état asymptotique, comme une visée jamais véritablement effectuée, comme un horizon qui ne devra jamais être atteint. Dans cette perspective, la sortie réelle de l'esclavage devra supposer la sortie de l'amnésie culturelle.

L'esclavage étant un état-limite, un indicible, les possibilités de sortir de cette condition ont été recherchées dans des lieux comme la religion et dans les arts, dont en particulier la musique et la danse. La première phase de révolte des esclaves a été justement le travail de reconstruction/ reconstitution d'une culture propre à travers la recréation du temps et de l'espace, et donc d'abord à travers un nouveau rapport à l'origine, c'est-à-dire à l'Afrique perdue. Le premier mouvement a été le culte des morts comme négation de la mort sociale, car ce culte relie l'individu aux morts récents, aux ancêtres, puis aux divinités africaines. Ainsi une chaîne de signifiants formant langage symbolique est mise en route, qui va à l'encontre de l'amnésie culturelle. L'attachement passionné des esclaves à la religion doit être compris comme la quête d'un ailleurs en vue de s'évader de l'assujettissement au maître, ou à tout le moins en vue de relativiser le pouvoir du maître. Finalement les esclaves finissent par créer une nouvelle culture qui émerge comme la résultante d'un mariage/arrimage de signifiants captés du système religieux imposé (le christianisme) et de signifiants repris des croyances africaines. Peu à peu s'est construit un processus de reconnaissance mutuelle, de production d'un nouveau lien social audelà des ethnies différentes et dispersées. Les pratiques de rébellion et de marronnage ou fuite hors des plantations et des ateliers n'auraient pas eu d'efficace hors de l'action quotidienne de reconstitution d'un ordre symbolique qui donne aux esclaves la possibilité de réintégrer de manière durable la condition de dignité d'être humain. Le succès de l'insurrection générale des esclaves en 1791 suppose que les esclaves ont eu conscience de constituer une collectivité qui a des intérêts communs et ont acquis un sens très fort de la solidarité. 


\section{Les difficultés du nouvel État indépendant}

\section{Retour à la table des matières}

La révolution haïtienne connaîtra cependant une série de difficultés et d'obstacles pour se stabiliser, dont nous aimerions rendre compte au moins de manière sommaire avant d'aborder l'interrogation sur sa portée universelle.

[61] Au niveau interne tout d'abord, on s'aperçoit que sur le plan politique, l'expérience est inédite: pour la première fois des esclaves sortis par eux-mêmes de l'esclavage bâtissent un nouvel État. Que le pays ait connu trois régimes successifs (empire, république, royauté), parfois concomitants, a suscité parfois des jugements hâtifs sur une propension des Noirs au despotisme qui va à l'encontre du rêve de liberté qui animait les masses d'esclaves dans la guerre pour l'indépendance. Sans aucun doute, ce n'est pas encore le projet d'établissement d'une démocratie qui est à l'œuvre dans les premières décennies de l'indépendance, mais à cette époque l'obsession des premiers chefs d'état haïtiens est de rejoindre les modèles européens de pouvoir. Au niveau culturel et religieux, les prêtres de la Constitution civile du clergé sont envoyés par l'abbé Grégoire qui soutient activement la révolution haïtienne, mais l'État haïtien restera préoccupé d'obtenir à l'instar de Napoléon un concordat avec le Vatican pour faire de l'Église catholique l'institution religieuse officielle du pays. Soulignons toutefois qu'encore dans la première Constitution en 1805 il est écrit que tous les cultes sont permis. Cependant il ne pouvait être question que l'État puisse reconnaître par exemple le vodou comme une religion à part entière et comme un dispositif de pratiques admissibles dans l'espace public. Les difficultés rencontrées par le nouvel État indépendant n'ont rien à voir avec une quelconque précocité de la révolution, il fallait à la fois maintenir une orientation commune aux divers 
groupes sociaux, lutter contre la hiérarchie raciale qui avait été à la base de l'institution esclavagiste, construire partout à travers le pays des fortifications pour prévenir le retour du colonisateur, relancer la production économique, organiser la distribution des terres, protéger le pays contre le retour des Français, et par-dessus tout montrer que l'État est un État comme tous les autres par la langue, la religion, l'éducation, la forme de gouvernement, etc.

Toutes ces difficultés sont inscrites dans un contexte qui les rend difficilement surmontables. Ce contexte est celui de la stratégie des puissances coloniales décidées à accomplir une nouvelle conquête du monde. Je reprends ici en particulier les données apportées par le regretté Yves Benot dans son ouvrage sur La Démence coloniale sous Napoléon ${ }^{7}$. L'expédition de Napoléon en 1802 visait, on le sait, le rétablissement de l'esclavage alors que Saint Domingue, sous le gouvernement de Toussaint Louverture depuis 1801, est doté d'une constitution consacrant la suppression de l'esclavage réalisée depuis 1791. Mais c'est toute une nouvelle série de projets coloniaux qui est mise en marche à la même époque : expéditions à Alger, en Inde, en Égypte. Benot parle d'une véritable démangeaison coloniale qui accapare Napoléon. [62] Se trouve posé déjà ce qui restera déterminant tout au long du XIX siècle et jusque dans toute la deuxième moitié du $X X^{e}$ siècle. La création des lois racistes (interdiction pour les Noirs d'entrer et de séjourner en France, interdiction des mariages mixtes), puis les lois d'exception pour les colonies, ce sont là des orientations qui ont une portée durable dans la métropole pour soutenir le principe de la supériorité de la civilisation européenne et faire accepter le partage du monde entre pays colonisateur et pays colonisé. C'est donc à un moment où l'idéologie raciste connaît un nouvel essor qu'Haïti fait ses premiers pas comme État indépendant. Le pays devra nécessairement vivre sous la menace constante du retour de la France coloniale et esclavagiste. Le 17 avril 1825, Charles $X$ envoie une expédition de 14 bâtiments dans la rade de la capitale avec une ordonnance concernant

7 Y. Benot, La Démence coloniale sous Napoléon, La Découverte, Paris, 1992. 
une indemnité de 150 millions de francs pour la reconnaissance de l'indépendance d'Haïti.

Il est intéressant de noter que tous les vieux schémas racistes de la période esclavagiste sont repris dans la plupart des rapports et discours qui prétendent fournir une interprétation de la révolution haïtienne. Non seulement on parvenait ainsi à la banaliser, mais aussi à montrer à force de caricature 8 que les Noirs sont incapables de se diriger par eux-mêmes et qu'ils ne cesseront de voir dans la colonisation elle-même un bienfait pour eux. Dans les rapports sur les causes des troubles de Saint Domingue à partir de l'insurrection générale de 1791 , on prétend que ce serait sous l'impulsion de puissances étrangères que cette insurrection se serait réalisée. Plus tard, Talleyrand déclarera suite au massacre de Français ordonné par Dessalines en 1805 (en représailles contre les massacres organisés par le général Rochambeau): «Il est devenu nécessaire de renouveler les représentations déjà adressées au gouvernement fédéral et d'insister de nouveau auprès de lui pour qu'il adopte enfin des mesures sévères et propres à prévenir de semblables communications entre une nation policée et des peuplades sauvages qui, par leurs mours féroces et leurs usages barbares, sont devenues étrangères au système de la civilisation... Quel est en effet le résultat de ces rapports commerciaux? De fournir à des hommes atroces les moyens de perpétuer leurs excès, de leur donner le pouvoir de signaler par de nouvelles cruautés une haine constante et générale pour tous les hommes que la naissance, les mœurs distinguent d'eux... L'existence d'une peuplade nègre armée et occupant les lieux qu'elle a souillés par les actes les plus criminels est un spectacle horrible pour toutes les nations blanches... ${ }^{9}$ » On peut dire que le ton est donné pour le déploiement au niveau de l'histoire et de l'anthropologie au XIX siècle et jusqu'à l'époque de la deuxième guer-

8 Voir l'ouvrage de C. H. Middelanis, Imperialen Gegenwelten. Haiti in den franzosichen Text-und Bildmedien, Vervuet Verlag, Frankfurt, 1996, qui est une remarquable recherche très approfondie sur les textes et les caricatures (publiés en Europe) visant à banaliser la révolution haïtienne pendant le XIXe siècle.

9 Y. Benot, La Démence coloniale sous Napoléon, op. cit., p.123. 
re [63] mondiale pour le succès de l'opposition barbare/civilisé 10 comme grille de justification du colonialisme. Essayons cependant de voir dans quelle mesure la révolution haïtienne représentera une avancée dans une vision postcoloniale de l'histoire.

\section{La révolution haïtienne et la sortie de la vision coloniale de l'histoire}

\section{Retour à la table des matières}

Récemment Michel Rolf Trouillot, historien haïtien, a soutenu dans un ouvrage intitulé Silencing the past 11 l'idée que la révolution haïtienne a été un événement inouii, imprévisible pour le colon, mais aussi incompréhensible et impensable à proprement parler pour les philosophes et les historiens pendant longtemps, tant les préjugés sur une prétendue incapacité des Noirs à accéder au sens de la liberté étaient forts. Il fallait donc taire la révolution haïtienne parce qu'elle n'était pas intégrable dans les dispositifs traditionnels de pensée sur le monde et l'histoire. Un problème épistémologique se trouverait à la racine de cette difficulté de rendre compte du caractère abrupt de l'événement insurrectionnel de 1791. Il y aurait donc une tâche de reproduction de la pensée coloniale comme telle à travers l'interprétation qui est produite traditionnellement dans I'historiographie occidentale de la révolution haïtienne. L'œuvre de David Brion Davis 12 a ouvert le chemin pour l'étude critique de la pensée de l'esclavage et des luttes entreprises pour son abolition dans la

10 Cf. notre ouvrage intitulé Le Barbare imaginaire, Éd. du Cerf, coll. « Sciences humaines et religions», Paris, 1988 (en cours de réédition aux éditions de la Maison des Sciences de l'Homme (2007).

11 M.R. Trouillot, Silencing the past, Power and the production of history, Beacon Press, Boston, 1995.

12 D.B. Davis, The problem of Slavery in Western Culture, Cornell University Press, Ithaca and London, 3e éd., 1969. Voir aussi The problem of Slavery in the Age of revolution, 1770-1823, Cornell University Press, New York, Ithaca, 1975. 
culture occidentale. Cette œuvre souligne en particulier comment l'esclavage a été davantage compris au XVII siècle et à l'époque des Lumières comme une métaphore de la domination de l'homme par l'homme que comme un système précis dans lequel le monde occidental a été engagé massivement pour son propre développement. Les philosophes du contrat social (Grotius, Pufendorf et Locke notamment) n'ont pas toujours perçu une incompatibilité entre leurs théories et la pratique de l'esclavage des Noirs dans les Amériques. De même Eugene Genovese 13 a tenté avec succès de décrire le monde qu'ont bâti les esclaves et sa signification pour l'humanité universelle. On ne saurait cependant nier que des valeurs universelles ont été affirmées par les Lumières, en dépit du caractère contradictoire des énoncés sur la liberté et l'égalité. Montesquieu, Diderot et l'Abbé Raynal par exemple ont clairement désapprouvé l'esclavage comme contraire aux lois naturelles. Que Rousseau ait pu déclarer que «l'homme est né libre et que partout il est dans les fers», ou encore que ce qui existe ce ne sont pas des races ou des groupes humains séparés, mais l'espèce humaine, il est clair que l'universel [64] affirmé peut être interprété comme un horizon susceptible d'être un régulateur dans l'histoire des rapports entre les peuples. En revanche, la vision qu'on a généralement en Europe des cultures africaines et la dissimulation des révoltes des esclaves dans l'opinion publique ont été des facteurs qui ont conduit à la proposition d'une abolition graduelle de l'esclavage.

Il y a une certaine réception en Allemagne et en Angleterre de la révolution haïtienne qu'il faudra bien approfondir. On trouvera dans les travaux de David Geggus ${ }^{14}$ des données nouvelles qui rompent avec la folklorisation de la révolution haïtienne qu'on découvre facilement en France au XIXe siècle. Mais retenons pour le moment l'interrogation récente entreprise par Susan Buck-Morss sur la dialectique du maître et de l'esclave qui est l'épine dorsale de la Phénoménologie de l'Esprit. Dans cette œuvre rédigée à Iéna en 1805-1806 Hegel semble appuyer

13 E. Genovese, The World the Slaves made, Pantheon Books, New York, 1974.

14 D. Geggus (sd), The impact of the Haitian revolution in the atlantic World, University of South Carolina Press, 2001. 
l'idée de lutte à mort de l'esclave pour la liberté sur les informations qu'il avait concernant la révolution haïtienne: « Et c'est seulement par la mise en jeu de la vie qu'est ainsi éprouvée et avérée la liberté. L'individu qui n'a pas mis sa vie en jeu peut, certes, être reconnu comme personne: mais il n'est pas parvenu à la vérité de cette reconnaissance, comme étant celle d'une conscience de soi autonome». Hegel semble ainsi rompre avec la thèse de la conquête de la subjectivité par le travail qu'il soutenait dans Le système de la vie éthique (1803). Pour Susan Buck-Morss 15, Hegel, qui lisait régulièrement les nouvelles de Saint Domingue-Haïti dans la revue Minerve en 1804-1805, soutenait que « le monde avait les yeux tournés vers Saint Domingue. » La revue Minerve était lue par Goethe, Schelling et Schiller. Sans donc citer explicitement la révolution française, ni la révolution haïtienne, Hegel défendait le caractère universel du désir de liberté.

Dans tous les cas, ce que la dialectique du maître et de l'esclave donne à penser, c'est la nécessité actuelle d'un approfondissement de la signification de la révolution haïtienne pour l'histoire universelle. Le phénomène n'est pas un appendice de la révolution française, ni sa simple conséquence. C'est même la révolution haïtienne qui permet de comprendre toute la portée de la déclaration universelle des droits de I'homme et du citoyen. Aimé Césaire et Alejo Carpentier l'ont fait saisir mais de manière récente dans la deuxième moitié du $X X^{e}$ siècle, rompant ainsi avec cette folklorisation de la révolution haïtienne.

C'est désormais l'Occident qui devrait s'efforcer de rattraper la révolution haïtienne. Mais pour cela il convient de sortir de la vision coloniale de l'histoire, comme histoire du monde occidental qui se déploie pendant que les autres sociétés qui ont connu l'esclavage et la [65] colonisation tardent à entrer dans la modernité et dans la rationalité. La révolution haïtienne a dès le début du XIX siècle annoncé ce qui allait être l'orientation normative pour tous les peuples, dans la me-

15 S. Buck-Morss, Hegel et Haïti, trad. de l'anglais par N. Ségol, éd. Lignes-Leo Scheer, Paris, 2006 ; voir également, du même auteur, Les Lumières, l'esclavage, la colonisation, La Découverte, Paris, 2005. 
sure où la sortie de l'ère de la domination raciale, esclavagiste et coloniale est la tâche encore à accomplir au niveau de la pensée comme au niveau de la réalité du monde. De fait la révolution haïtienne avait renforcé les tendances aux insurrections et révoltes d'esclaves au XIX siècle à travers la Caraïbe et les Amériques en général, puis elle a effectivement réussi à exporter en Amérique latine l'idée de lutte anticoloniale et anti-esclavagiste pour le Venezuela notamment à travers Miranda et Bolivar. L'on ne saurait donc faire de la révolution haïtienne un événement qui resterait confiné seulement dans la mémoire des anciens esclaves. Le phénomène de l'esclavage outre-Atlantique appartient également à la mémoire de l'Occident et n'est point non plus un reste ni un accident de la modernité. Il mérite d'être compris comme un événement constitutif de la modernité occidentale, comme la face qu'elle cherche en vain à cacher et qui sans cesse revient la hanter. 


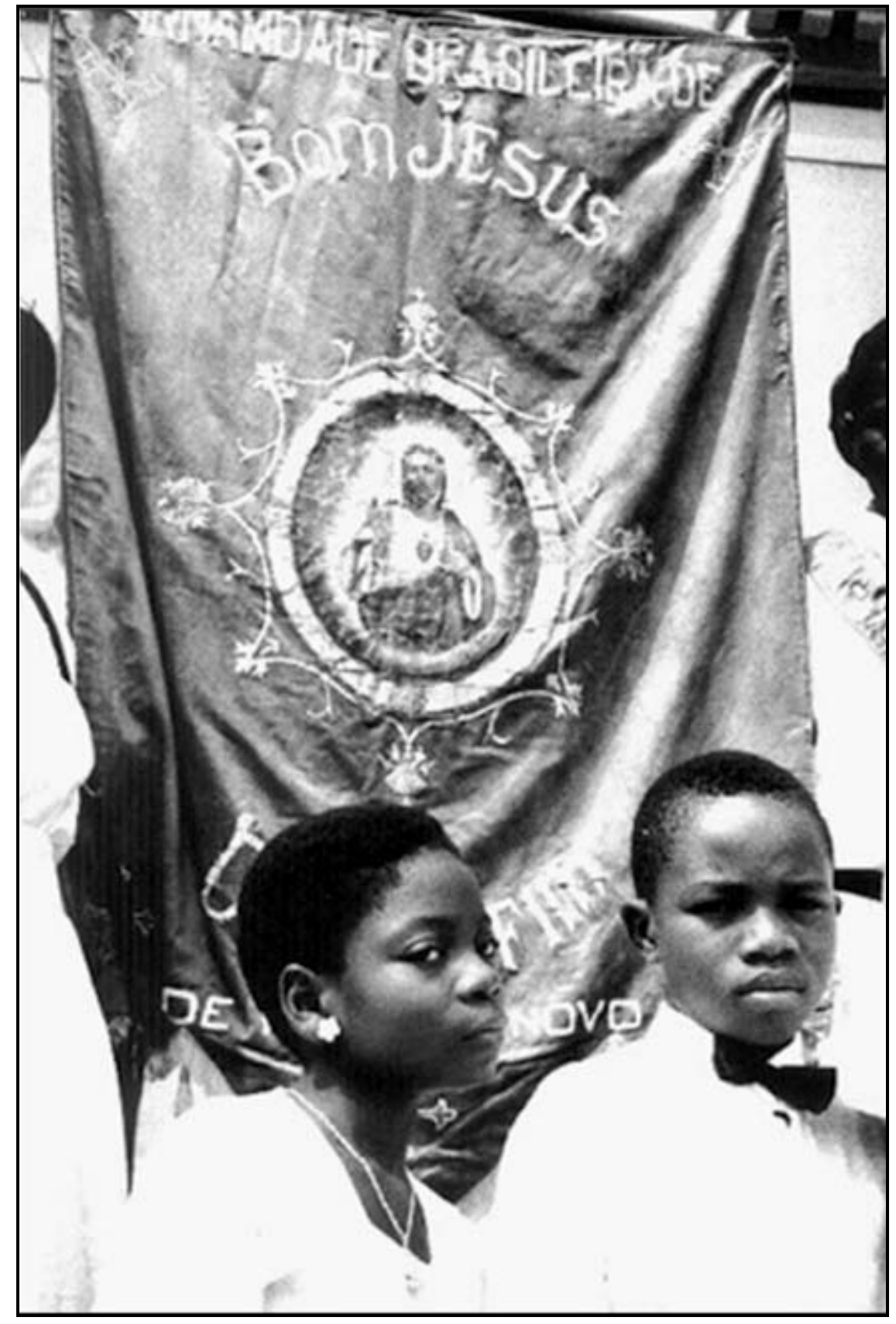

Milton Guran, Agoudas du Bénin. @ Milton Guran.

Fin du texte 